\title{
Síndrome de Noonan: do Fenótipo à Terapêutica com Hormônio de Crescimento
}

revisão

\author{
Alexsandra C. Malaquias \\ LIZE V. FERREIRA \\ SILVIA C. SOUZA \\ IVO J. P. ARNHOLD \\ BERENICE B. MENDONÇA \\ AleXANDER A. L. JoRge
}

Unidade de Endocrinologia do Desenvolvimento, Laboratório de Hormônios e Genética Molecular

LIM/42, Disciplina de

Endocrinologia da Faculdade de Medicina da Universidade de São Paulo (FMUSP), SP, Brasil.
RESUMO

A síndrome de Noonan (SN) é uma síndrome genética comum que constitui importante diagnóstico diferencial em pacientes com baixa estatura, atraso puberal ou criptorquidia. A SN apresenta grande variabilidade fenotípica e é caracterizada principalmente por dismorfismo facial, cardiopatia congênita e baixa estatura. A herança é autossômica dominante com penetrância completa. O diagnóstico é clínico, com base em critérios propostos por van der Burgt, em 1994. Recentemente, diversos genes envolvidos na via de sinalização RAS-MAPK foram identificados como causadores da SN: PTPN11, KRAS, SOS1, RAF1 e MEK1. O tratamento com hormônio de crescimento (hrGH) é proposto para corrigir a baixa estatura observada nestes pacientes. Estudos recentes apontam que pacientes com SN por mutações no gene PTPN11 apresentam pior resposta ao tratamento com hrGH quando comparado com pacientes sem mutações no PTPN11. Este artigo revisará os aspectos clínicos, moleculares e do tratamento da baixa estatura de crianças com SN com hrGH. (Arq Bras Endocrinol Metab 2008; 52/5:800-808)

Descritores: Síndrome de Noonan/genética; Síndrome de Noonan/terapia; Hormônio do crescimento/uso terapêutico; Insuficiência de crescimento; Genética médica

\section{ABSTRACT}

Noonan Syndrome: from Phenotype to Growth Hormone Therapy.

Noonan Syndrome (NS) is one of the most common genetic syndromes and it is an important differential diagnosis in children with short stature, delayed puberty and cryptorchidism. NS is characterized by dysmorphic facial features, congenital heart defects and short stature, but there is a great variability in phenotype. NS may occur in a pattern consistent with autosomal dominant inheritance with almost complete penetrance. The diagnosis is based on a clinical score system proposed by van der Burgt e cols. in 1994. In recent years, germline mutations in the components of RAS-MAPK (mitogen activated protein kinase) pathway have been shown to be involved in the pathogenesis of NS. Mutations in PTPN11, KRAS, SOS1, RAF1 e MEK1 can explain $60-70 \%$ of NS molecular cause. Growth hormone therapy is proposed to correct the short stature observed in these patients. Recent studies suggest that the presence of PTPN11 mutations in patients with NS indicates a reduced growth response to short-term hrGH treatment. In this article, it is reviewed clinical and molecular aspects of NS and hrGH treatment for short stature. (Arq Bras Endocrinol Metab 2008; 52/5:800-808)

Keywords: Noonan syndrome/genetics; Noonan syndrome/therapy; Growth hormone/pharmacology; Growth hormone/therapeutic use; Failure to thrive genetics; medical 


\section{INTRODUÇÃO}

A Síndrome de Noonan (SN; OMIM 163950) é uma das mais freqüentes síndromes de herança mendeliana, com incidência estimada entre 1:1.000 a 1:2.500 nascidos vivos $(1,2)$. Esta síndrome foi inicialmente descrita em 1963 por Jacqueline Noonan, cardiologista pediátrica que relatou nove pacientes (três do sexo feminino) com estenose valvar pulmonar associada à baixa estatura, dismorfismo facial e retardo mental moderado (3). O termo síndrome de Noonan foi proposto posteriormente por Optiz, em 1965 (4).

A SN tem herança autossômica dominante, distribuição semelhante entre os sexos e fenótipo bastante variável (5). As crianças com SN freqüentemente procuram os endocrinologistas com queixa de baixa estatura, puberdade atrasada, e, no sexo masculino, também por criptorquidia. Esta revisão abordará os aspectos clínicos, moleculares e do tratamento da baixa estatura com hrGH de crianças com SN.

\section{FENÓTIPO DA SÍNDROME DE NOOMAN}

\section{Características faciais}

A face típica da SN é caracterizada por sua forma triangular, hipertelorismo ocular, ptose palpebral, fissura palpebral externa desviada para baixo, implantação baixa e rotação incompleta do pavilhão auricular, com espessamento da hélice auricular, micrognatia e pescoço curto ou alado (Figura 1). As características faciais típicas da SN podem estar presentes desde o nascimento.

Ao longo dos dois primeiros anos de vida, a cabeça adquire um aspecto turricefálico com eminência malar achatada e estreitamento bitemporal. Os olhos são proeminentes, arredondados e, ocasionalmente, observa-se hipertelorismo ou estrabismo. O filtro nasal é alargado, com a base do nariz rasa, narinas antevertidas e columela curta, conferindo ao nariz um aspecto de pêra. Durante a infância ocorre acentuação da ptose palpebral e a face progressivamente assume formato mais triangular, à medida que o queixo se alonga. O pescoço é aparentemente curto, mas observa-se menos excesso de pele se comparado ao período neonatal. $\mathrm{Na}$ adolescência, o pescoço cresce e o trapézio alado fica mais evidente. As características faciais atenuam-se com o avançar da idade, dificultando o reconhecimento da SN na idade adulta (6). Adultos com SN apresentam pregas nasolabiais proemi-

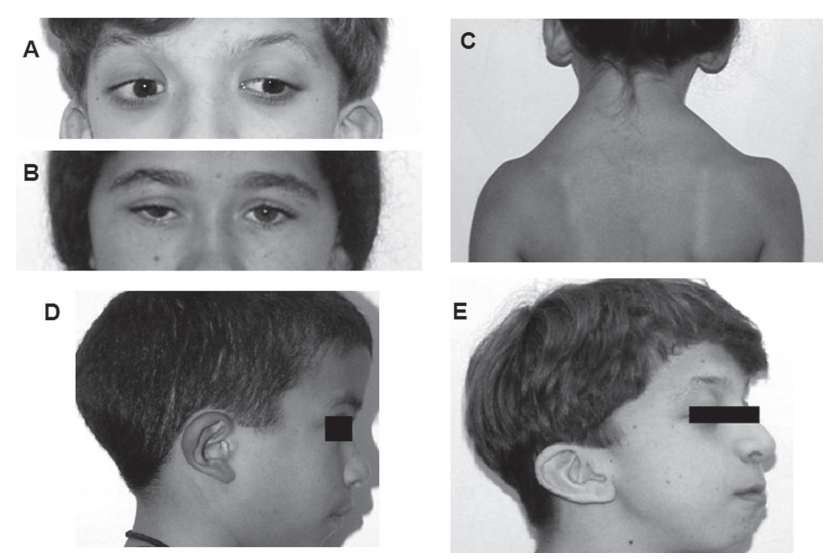

Figura 1. Características clínicas de pacientes com síndrome de Noonan: A) epicanto; B) ptose palpebral; C) pescoço alado e implantação baixa de cabelos; D) espessamento de hélice auricular; E) baixa implantação e rotação incompleta do pavilhão auricular.

nentes, implantação anterior do cabelo alta e rugas faciais principalmente na fronte $(7,8)$.

\section{Crescimento}

A baixa estatura de início pós-natal é uma das características clínicas mais freqüentemente observadas na $\mathrm{SN}$, afetando cerca de $70 \%$ a $83 \%$ dos pacientes $(8,9)$. Durante a infância observa-se crescimento paralelo à curva de referência (10) (Figura 2) com escore de desviospadrão de altura em média de -3 (11-14), observando-se atraso de um a dois anos na idade óssea. A intensidade da baixa estatura não é explicada pela presença de defeitos cardíacos ou pela dificuldade alimentar observada nestes pacientes na infância (15). Estudos na população holandesa ou alemã verificaram que a estatura média de indivíduos adultos com SN não tratados com hrGH é de, respectivamente, 161,0 e 162,5 $\mathrm{cm}$ no sexo masculino e de 150,5 e $152,7 \mathrm{~cm}$ no sexo feminino, correspondendo ao escore de desvio-padrão da altura final ( $\mathrm{Z}$ da altura final) de $-2,5$ a $-2,1$.

A etiologia da baixa estatura na $\mathrm{SN}$ ainda não está esclarecida. Estudos realizados avaliando o eixo GH/ IGF-1 em pacientes com SN apresentaram resultados contraditórios. A resposta aos testes de estímulo de secreção de GH é usualmente normal (9). A avaliação da 
secreção espontânea de $\mathrm{GH}$ em crianças com $\mathrm{SN}$, por meio do ritmo de $\mathrm{GH}$, mostra secreção diminuída em um estudo (16) e normal com tendência a ser elevada em dois outros $(17,18)$. Os níveis de IGF-1 estão abaixo do normal ou no limite inferior da normalidade na maioria dos pacientes. A presença de IGF-l baixo e a secreção de GH normal ou elevada sugere que um grau de insensibilidade ao GH possa ter papel na baixa estatura característica dessa síndrome $(14,18,19)$.

\section{Alterações cardíacas}

Os defeitos cardíacos congênitos são observados em $62 \%$ a $90 \%$ dos pacientes na maioria das casuísticas. Provavelmente esta freqüência é superestimada em virtude da seleção de pacientes, principalmente provenientes de serviços de cardiologia, na maioria dos estudos. Em nosso grupo, em que os pacientes com SN são encaminhados principalmente pela baixa estatura, a frequiência de defeitos cardíacos congênitos foi de $30 \%$ (9). A estenose valvar pulmonar é a lesão cardíaca mais comum na SN, estando presente em $54 \%$ dos pacientes, seguido por miocardiopatia hipertrófica e defeitos do septo atrial, ambos presentes em 18\% dos pacientes. Os defeitos no septo ventricular (11\%), a insuficiência mitral $(6 \%)$, a coartação de aorta (4\%), a estenose aórtica (4\%) e a persistência do canal arterial são também descritos em pacientes com SN (20). O eletrocardiograma típico da $\mathrm{SN}$ mostra complexos QRS alargados com predomínio de padrão negativo nas derivações precordiais à esquerda. Pode-se obser-

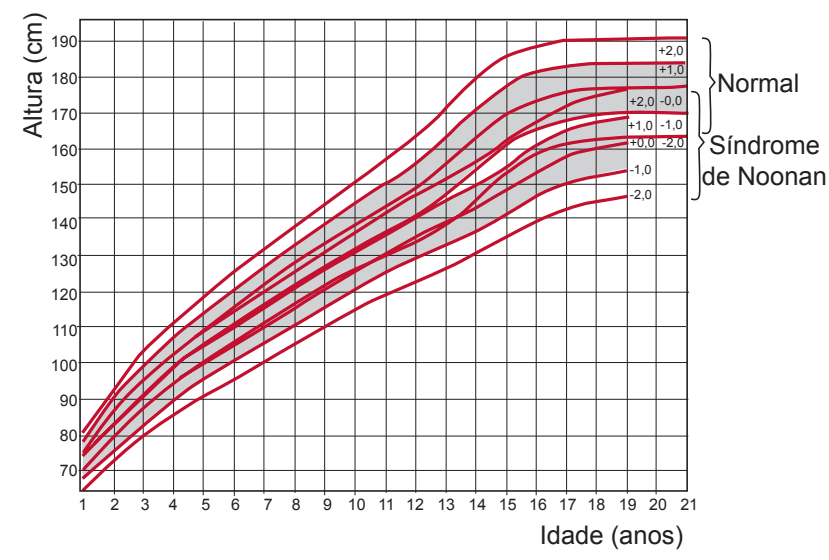

Figura 2. Gráfico de crescimento da altura por idade para crianças normais e para crianças com síndrome de Noonan na população alemã (10). var também desvio de eixo para a esquerda e ondas $Q$ gigantes (6).

\section{Alterações esqueléticas}

Em torno de 3 a 4 anos de idade, as deformidades torácicas tornam-se evidentes, com pectus carinatum e/ou pectus excavatum. O tórax é largo, lembrando o formato de um escudo com hipertelorismo mamário. Outras anormalidades esqueléticas incluem cúbito valgo, clinobraquidactilia, escoliose/cifose e má oclusão dentária $(7,8)$.

\section{Alterações linfáticas}

As alterações linfáticas ocorrem em menos de $20 \%$ dos pacientes e são decorrentes de aplasia, hipoplasia ou displasia dos vasos linfáticos, levando à linfedema generalizado ou periférico, linfangiectasia pulmonar ou intestinal, hidropsia fetal e higroma cístico $(7,8)$. O higroma cístico observado no período pré-natal se mantém como excesso de pele na região da nuca no recém-nascido. Criptorquidia, hipertelorismo mamário, baixa implantação com rotação incompleta das orelhas, hipertelorismo ocular e desvio do ângulo ocular externo para baixo são decorrentes da migração anormal de tecidos ou órgãos causados pelo edema linfático $(6)$.

\section{Outras alterações}

O atraso no desenvolvimento neuropsicomotor é observado em $40 \%$ a $70 \%$ dos pacientes com SN, porém o retardo mental incapacitante é incomum. A hipotonia muscular muitas vezes colabora para o retardo no desenvolvimento motor (6). Grau moderado de retardo mental é observado em $15 \%$ a $35 \%$ dos pacientes e dificuldade de aprendizado que exija acompanhamento especial ocorre em $26 \%$ dos casos. Entretanto, a maioria dos indivíduos com SN (85\%) apresenta boa qualidade de vida e integração social $(6,21)$.

Mais da metade dos pacientes do sexo masculino apresenta criptorquidia uni ou bilateral (6-8). A puberdade ocorre espontaneamente, mas freqüentemente é atrasada e de curta duração, com rápido avanço da idade óssea no período intrapúbere, por mecanismo ainda indefinido. A fertilidade feminina é preservada (8) e o comprometimento da fertilidade masculina está provavelmente associado à criptorquidia.

Em mais de 55\% dos pacientes observa-se história de equimoses espontâneas ou tendência a sangramen- 
to prolongado. Alterações hematológicas, como deficiência de fatores VIII, XI e XII, trombocitopenia e defeitos na função plaquetária são encontrados em $20 \%$ dos casos, porém, eventos hemorrágicos graves são $\operatorname{raros}(3 \%)(6-8)$ (Tabela 1$)$.

\section{DIAGNÓSTICO DA SN}

O diagnóstico da $\mathrm{SN}$ deve ser fundamentado nos achados clínicos. A SN freqüentemente é lembrada como diagnóstico diferencial em pacientes com face típica e/ou com estenose pulmonar. Mas o diagnóstico da $\mathrm{SN}$ pode ser difícil, principalmente por causa da grande variabilidade fenotípica, com a presença de pacientes com características faciais discretas e/ou na ausência de malformações cardíacas. A atenuação dos traços faciais nos indivíduos adultos é outro fator que dificulta o diagnóstico após a infầncia. Em 1981, Duncan e cols. propuseram um sistema de escore para o diagnóstico da SN com base em 26 itens encontrados comumente nestes pacientes (22). A complexidade deste primeiro sistema de escore dificultou o seu uso rotineiro. Em 1994, van der Burgt e cols. propuseram um sistema simples e eficiente com base na pontuação de critérios maiores e menores que considera a variabilidade clínica presente na SN (Tabela 2) (23).

Tabela 1. Anormalidades fenotípicas associadas à SN. As freqüências são mostradas entre parênteses $(1,7,22)$.

\begin{tabular}{|c|c|}
\hline Características & Sinais \\
\hline Herança & Autossômica dominante \\
\hline Crescimento & $\begin{array}{l}\text { Baixa estatura (pós-natal) (50\%-80\%) } \\
\text { Failure to thrive na infância (40\%) }\end{array}$ \\
\hline Cabeça e pescoço & $\begin{array}{l}\text { Face triangular progressiva com a idade } \\
\text { Anormalidades de pavilhão auricular (44\%-90\%): baixa implantação, rotação } \\
\text { incompleta, espessamento de hélice } \\
\text { Anormalidades oculares (95\%): ptose, hipertelorismo, epicanto, fissuras palpebrais } \\
\text { voltadas para baixo, estrabismo, proptose, miopia e nistagmo } \\
\text { Filtrum nasal alargado (95\%) } \\
\text { Palato ogival (45\%-34\%) } \\
\text { Micrognatia (22\%) } \\
\text { Má oclusão dentária (35\%) } \\
\text { Baixa implantação de cabelos posteriormente (32\%) } \\
\text { Anormalidades cervicais (95\%): pterygium colli, pescoço curto ou alado. }\end{array}$ \\
\hline Cardiovascular & $\begin{array}{l}\text { Cardiopatias congênitas (50\%-75\%): estenose valvar pulmonar (50\%), miocardiopatia } \\
\text { hipertrófica (10\%), defeito de septo atrial (10\%) e outros (estenose aórtica, defeitos } \\
\text { de septo ventricular e insuficiência mitral) } \\
\text { Eletrocardiograma com desvio de eixo para esquerda e padrão negativo nas } \\
\text { derivações precordiais à esquerda }\end{array}$ \\
\hline Tórax & $\begin{array}{l}\text { Anormalidades torácicas (53\%-70\%): pectus carinatum superiormente e/ou pectus } \\
\text { excavatum inferiormente, tórax alargado e outras deformidades }\end{array}$ \\
\hline Geniturinário & $\begin{array}{l}\text { Criptorquidia (60\%-69\%) } \\
\text { Atraso puberal }\end{array}$ \\
\hline Esqueleto & $\begin{array}{l}\text { Anormalidades da coluna vertebral (25\%) } \\
\text { Cúbito valgo (47\%) } \\
\text { Anormalidades das mãos: clinodactilia/braquidactilia }\end{array}$ \\
\hline Neurológico & $\begin{array}{l}\text { Atraso do desenvolvimento motor (26\%), dificuldade de aprendizado (15\%) e } \\
\text { atraso de linguagem (20\%) } \\
\text { Retardo mental leve (25\%-35\%) }\end{array}$ \\
\hline Hematológico & $\begin{array}{l}\text { Anormalidades de sangramento ( } 20 \% \text { ), incluindo deficiência de fator XI ou XII, doença de } \\
\text { von Willebrand, disfunção plaquetária e leucemia (em especial leucemia mielomonocítica } \\
\text { juvenil - JMML) }\end{array}$ \\
\hline Outros & Linfedema periférico, esplenomegalia, surdez \\
\hline
\end{tabular}


Tabela 2. Critérios de van der Burgt e cols. para diagnóstico da síndrome de Noonan (23).

\begin{tabular}{|c|c|c|c|}
\hline \multirow[t]{2}{*}{ Características } & & \multicolumn{2}{|c|}{ Critérios } \\
\hline & & Maiores & Menores \\
\hline Faciais & & Típica & Sugestiva \\
\hline Cardíacas & & $\begin{array}{l}\text { Estenose valvar pulmonar } \\
\text { Miocardiopatia hipertrófica }\end{array}$ & Outras \\
\hline Altura & & $<3^{\circ}$ percentil & $<10^{\circ}$ percentil \\
\hline Torácicas & & $\begin{array}{c}\text { Pectus carinatum } \\
\text { e/ou pectus excavatum }\end{array}$ & Alargado \\
\hline História familiar & & $\begin{array}{c}\text { Parente de } 1^{\circ} \text { grau com } \\
\text { diagnóstico de SN }\end{array}$ & $\begin{array}{c}\text { Parente de } 1^{\circ} \text { grau } \\
\text { sugestivo de SN }\end{array}$ \\
\hline Outros & $\begin{array}{l}\text { Retardo mental } \\
\text { Criptorquidia } \\
\text { Displasia linfática }\end{array}$ & Todos os três & Qualquer \\
\hline
\end{tabular}

Face típica = face triangular, fenda palpebral oblíqua com o ângulo esterno voltado para baixo, hipertelorismo ocular, ptose palpebral, pavilhão auricular malformado e de implantação baixa, micrognatia, pescoço alado.

\section{Diagnóstico de SN}

Face típica + um outro critério maior ou dois menores.

Face sugestiva + dois outros critérios maiores ou três menores.

Na década de 1990, estudos de linkage analysis permitiram a localização de uma região no braço longo do cromossomo 12 contendo o gene causador dessa síndrome $(24,25)$. Em 2001, Tartaglia e cols. demonstraram em pacientes com $\mathrm{SN}$ a presença de mutações missense em heterozigose no gene do PTPNII (protein tyrosinephosphatase non-receptor 11) localizado na região $12 \mathrm{q} 24.1$. Trabalhos subseqüentes consolidaram o PTPN1I como o gene mais freqüentemente responsá- vel pela SN e identificaram mutações em $29 \%$ a $60 \%$ dos casos $(9,26-30)$.

O PTPNII codifica a proteína tirosinofosfatase SHP-2 (Src Homology region 2-domain Phosphatase 2) universalmente expressa no organismo e importante na transdução do sinal da via MAPK (mitogen-activated protein kinase) (Figura 3) (31). As mutações do PTPNII descritas na SN resultam ganho de função da SHP-2 e aumentam a transdução do sinal intracelular

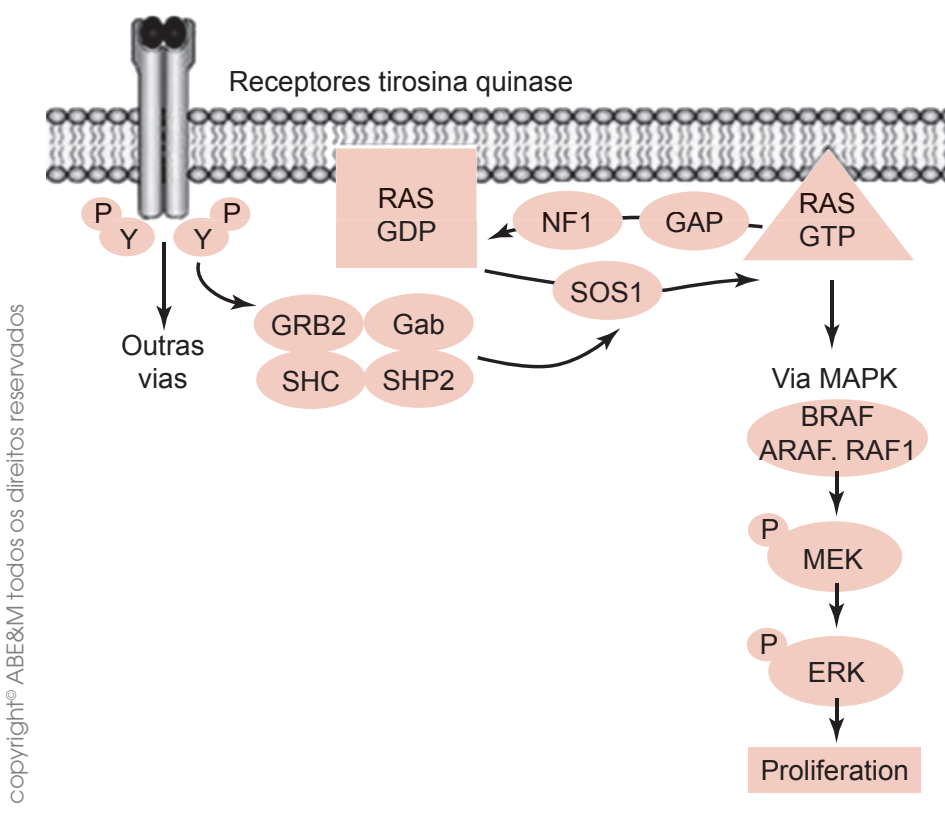

Figura 3. Representação da via RAS-MAPK. Adaptado de Schubbert e cols. (32). 
via MAPK (33). Para identificar a causa genética da SN em pacientes sem mutações no PTPNIl, foram estudados diversos outros genes que codificam proteínas envolvidas na via MAPK. Com esta estratégia novos genes envolvidos na patogênese da $\mathrm{SN}$ foram descritos: KRAS, SOS1, RAFl e MEKl (Tabela 3) (32, 34-40). As mutações associadas à $\mathrm{SN}$ observadas nestes genes também aumentam a transdução do sinal via MAPK.

Tabela 3. Genes envolvidos na etiologia da SN e a freqüência de mutações encontradas.

\begin{tabular}{lcc}
\hline Gene & Freqüência na SN & Referências \\
\hline PTPN11 & $29 \%-60 \%$ & $(9,21,26-30)$ \\
\hline SOS1 & $10 \%-17 \%$ & $(37-39)$ \\
RAF1 & $5 \%-20 \%$ & $(34,35)$ \\
KRAS & $1 \%-2,5 \%$ & $(41)$ \\
MEK1 & $4,3 \% *$ & $(40)$ \\
\hline
\end{tabular}

*Resultado expresso em relação a pacientes com SN sem mutação nos genes PTPN1 1 e SOS1.

A descrição de mutações nestes genes em pacientes com SN permitiu, pela primeira vez, a obtenção de marcadores genéticos para essa síndrome e demonstrou a acurácia do diagnóstico da SN utilizando os critérios clínicos de van der Burgt e cols. (23).

\section{DIAGNÓSTICO DIFERENCIAL}

Outras síndromes genéticas apresentam estigmas clínicos que se sobrepõem aos da $\mathrm{SN}$, entre elas a síndrome de Turner (ST) que acomete apenas pacientes do sexo feminino e que se encontra associada à disgenesia gonadal. O cariótipo diferencia a ST da SN pela presença de monossomia completa ou parcial dos cromossomos sexuais na ST, enquanto os pacientes com SN apresentam cariótipo normal.

Recentemente, mutações em outros genes na cascata da MAPK foram associadas a síndromes que apresentam traços clínicos semelhantes à $\mathrm{SN}$, como a síndrome de Costello (OMIM: 218040), a síndrome cardiofacecutânea (CFC, OMIM: 115150) e a síndrome de Leopard (lentigines múltiplas, anormalidades eletrocardiográficas de condução, hipertelorismo ocular, estenose pulmonar, anormalidades de genitália, retardo de crescimento, surdez (OMIM: 151100), sugerindo que estas síndromes Noonan-símile representam variações fenotípicas de uma mesma doença associada ao aumento da atividade da transdução do sinal via MAPK (32).

\section{TRATAMENTO COM HORMÔNIO DE CRESCIMENTO}

Diversos estudos demonstraram que o tratamento a curto prazo com GH recombinante humano (hrGH) é capaz de aumentar a velocidade de crescimento em 3 a $4 \mathrm{~cm} /$ ano em relação a velocidade de crescimento basal de crianças com SN $(13,14,18,19)$. A dose de hrGH utilizada na maioria dos trabalhos varia de 0,1 a 0,15 $\mathrm{U} / \mathrm{kg} /$ dia $(33$ a $50 \mu \mathrm{g} / \mathrm{kg} /$ dia $)$. A velocidade de crescimento no primeiro ano de tratamento de pacientes com SN pré-púberes $(7,9 \pm 1,6 \mathrm{~cm} / \mathrm{ano})$ (14) é inferior à observada em crianças com deficiência de $\mathrm{GH}$ (42) $(11,4 \pm 2,6 \mathrm{~cm} / \mathrm{ano})$ e semelhante à observada em pacientes com ST $(7,8 \pm 1,8 \mathrm{~cm} / \mathrm{ano})(43)$ ou crianças nascidas pequenas para a idade gestacional $(8,7$ $\pm 1,8 \mathrm{~cm} / \mathrm{ano})(44)$. Como em outras indicações de

Tabela 4. Diferença de resposta ao tratamento com hrGH de pacientes com síndrome de Noonan com ou sem mutação no gene PTPN1 1.

\begin{tabular}{|c|c|c|c|c|c|c|}
\hline \multirow[b]{2}{*}{ Mutação no PTPN1 1} & \multicolumn{2}{|c|}{ Binder e cols., 2005 (18) } & \multicolumn{2}{|c|}{ Ferreira e cols., 2005 (19) } & \multicolumn{2}{|c|}{ Limal e cols, 2006 (14) } \\
\hline & presente & ausente & presente & ausente & presente & ausente \\
\hline $\mathrm{n}$ & 8 & 3 & 7 & 7 & 15 & 10 \\
\hline Idade pré-tratamento (anos) & $7,4 \pm 2,2$ & $6,3 \pm 1,9$ & $12,9 \pm 4$ & $11,7 \pm 3$ & $10,4 \pm 3,1$ & $10,3 \pm 3,3$ \\
\hline Pré-púbere/púbere & $11: 0$ & $11: 0$ & $5: 2$ & $5: 2$ & $25: 0$ & $25: 0$ \\
\hline Dose de $\mathrm{GH}(\mu \mathrm{g} / \mathrm{kg} / \mathrm{dia})$ & $42 \pm 7$ & $50 \pm 8$ & $48 \pm 5$ & $46 \pm 7$ & 33 & 33 \\
\hline VC pré-tratamento (cm/ano) & NA & NA & $4,3 \pm 1,0$ & $3,9 \pm 1,0$ & $4,3 \pm 0,9$ & $5,2 \pm 1,4$ \\
\hline VC no $1^{\circ}$ ano (cm/ano) & NA & NA & $6,8 \pm 1,5$ & $7,6 \pm 1,9$ & $7,4 \pm 1,6$ & $8,5 \pm 1,7$ \\
\hline Z da altura inicial & $-3,5 \pm 0,7$ & $-3,8 \pm 0,1$ & $-3,6 \pm 1,0$ & $-3,4 \pm 1,0$ & $-3,5 \pm 0,9$ & $-3,0 \pm 0,8$ \\
\hline Incremento no $\mathrm{Z}$ da altura no $1^{\circ}$ ano & $0,7 \pm 0,2$ & $1,2 \pm 0,4$ & $0,3 \pm 0,4$ & $0,4 \pm 0,4$ & $0,5 \pm 1,2$ & $0,6 \pm 0,7$ \\
\hline
\end{tabular}


tratamento com hrGH, a velocidade de crescimento é melhor no primeiro ano de tratamento em relação aos anos subseqüentes $(12,13)$. Estudos recentes mostram que pacientes com SN com mutações no gene PTPNII apresentam menor resposta ao tratamento com hrGH $(14,18,19)$ (Tabela 4). Postula-se que a mutação na SHP-2 seria responsável por um estado de insensibilidade parcial do $\mathrm{GH}$ a nível pós-receptor $(14,18,19)$. Porém, ainda não existem estudos avaliando o efeito das mutações nos outros genes associados à $\mathrm{SN}$ sobre o crescimento e o eixo GH/IGF-1.

Poucos estudos avaliaram a altura final de pacientes com SN tratados com hrGH. Um desses trabalhos avaliou a altura adulta de 18 pacientes com SN tratados com hrGH na dose de 33 a $66 \mu \mathrm{g} / \mathrm{kg} /$ dia. O Z da altura final foi 1,7 DP maior que o $\mathrm{Z}$ da altura no início do tratamento, sendo semelhante nas duas doses testadas (45). Outro estudo relatou a altura adulta de 10 pacientes ingleses tratados em média por 5,3 anos na dose de $38 \mu \mathrm{g} /$ $\mathrm{kg} /$ dia. $\mathrm{O}$ ganho médio do $\mathrm{Z}$ de altura foi de $0,8 \pm 0,6$ (12). Não existem ainda estudos que analisem a influência do genótipo sobre a altura adulta de pacientes com $\mathrm{SN}$ tratados com hrGH. No nosso grupo, apenas três pacientes com mutação identificada no gene PTPNII atingiram a altura adulta após um mínimo de dois anos de tratamento com hrGH na dose de $50 \mu \mathrm{g} / \mathrm{kg} /$ dia. Estes pacientes apresentaram incremento de 1 a 1,9 no Z de altura em relação ao início do tratamento.

Complicações com o uso de $\mathrm{GH}$ não foram observadas na SN. A presença de cardiomiopatia hipertrófica (HCM) é uma contra-indicação ao uso de GH em razão de seus efeitos deletérios no músculo cardíaco, como visto na acromegalia. Cotterill e cols. (46) avaliaram o efeito do uso de GH durante 12 meses sobre o músculo cardíaco e não encontraram evidências de aumento na parede ventricular esquerda na vigência de tratamento. Alguns desses pacientes foram reavaliados após três anos de tratamento e não mostraram nenhuma evidência de aumento da parede ventricular esquerda. Não houve alteração do metabolismo lipídico e de carboidratos nesse mesmo estudo (13).

Outra preocupação do uso de hrGH em crianças com SN é o risco de desenvolvimento de leucemias. A ocorrência de leucemia em pacientes com SN encontra-se associada à presença de mutações específicas do gene PTPNII (T73I, Y62D, S502T, G503R e Q506P) e ainda não foi observada em pacientes com mutações nos outros genes associados à $\mathrm{SN}$. Diversos estudos que avaliaram o uso de hrGH em crianças com outros diagnósticos mostram que o tratamento com hrGH não aumenta o risco de leucemias, tanto em pacientes com $\mathrm{SN}(12,13,44)$ quanto em pacientes com outras indicações para o uso de hrGH (47).

$\mathrm{O}$ tratamento com hrGH em crianças com SN deve ser considerado experimental e por esta razão deve ser conduzido em centros médicos com experiência nesta síndrome. A decisão de tratar com hrGH deve avaliar os prós e os contras do tratamento da baixa estatura de cada criança e considerar o genótipo, uma vez que os pacientes positivos para mutação no gene do PTPNII são parcialmente resistentes ao $\mathrm{GH}$ e deveriam ser tratados com doses mais altas de hrGH.

\section{AGRADECIMENTOS}

Este trabalho obteve apoio da Fundação de Amparo à Pesquisa do Estado de São Paulo (FAPESP) Projeto Temático 05/04726-0 e Bolsa de Doutorado 07/59555-0 para A.C.M. E apoio do Conselho Nacional de Desenvolvimento Científico e Tecnológico (CNPq): 307951/06-5 para A.A.L.J., 301246/95-5 para B.B.M. e 300938/06-3 para I.J.P.A.

\section{Conflito de interesse}

Os autores declaram não ter conflito de interesse em relação ao presente artigo.

\section{REFERÊNCIAS}

1. Mendez HM, Opitz JM. Noonan syndrome: a review. Am J Med Genet. 1985;21:493-506.

2. Nora JJ, Nora AH, Sinha AK, Spangler RD, Lubs HA. The UIIrich-Noonan syndrome (Turner phenotype). Am J Dis Child. 1974;127:48-55

3. Noonan JA, Ehmke DA. Associated noncardiac malformations in children with congenital heart disease. J Pediatr. 1963; 63:468-70.

4. Optiz JM. Editorial comment: the Noonan syndrome. Am J Med Genet. 1985;21:515-8.

5. Allanson JE, Hall JG, Hughes HE, Preus M, Witt RD. Noonan syndrome: the changing phenotype. Am J Med Genet. 1985;21:507-14

6. van der Burgt I. Noonan syndrome. Orphanet J Rare Dis. 2007;2:4.

7. Allanson JE. Noonan syndrome. J Med Genet. 1987;24:9-13.

8. Noonan JA. Noonan syndrome and related disorders: alterations in growth and puberty. Rev Endocr Metab Disord. 2006;7:251-5.

9. Ferreira LV, Souza SC, Montenegro LR, Malaquias AC, Arnhold IJ, Mendonca BB, et al. Analysis of PTPN11 gene in idiopathic short stature children and Noonan syndrome patients. Clin Endocrinol (Oxf). 2008.

10. Ranke MB, Heidemann $P$, Knupfer $C$, Enders $H$, Schmaltz AA, Bierich JR. Noonan syndrome: growth and clinical manifestations in 144 cases. Eur J Pediatr. 1988;148:220-7. 
11. Romano AA, Blethen SL, Dana K, Noto RA. Growth hormone treatment in Noonan syndrome: the National Cooperative Growth Study experience. J Pediatr. 1996;128:S18-21.

12. Kirk JM, Betts PR, Butler GE, Donaldson MD, Dunger DB, Johnston DI, et al. Short stature in Noonan syndrome: response to growth hormone therapy. Arch Dis Child. 2001;84:440-3.

13. MacFarlane CE, Brown DC, Johnston LB, Patton MA, Dunger $\mathrm{DB}$, Savage MO, et al. Growth hormone therapy and growth in children with Noonan's syndrome: results of 3 years' followup. J Clin Endocrinol Metab. 2001;86:1953-6.

14. Limal JM, Parfait B, Cabrol S, Bonnet D, Leheup B, Lyonnet S, et al. Noonan syndrome: relationships between genotype, growth, and growth factors. J Clin Endocrinol Metab. 2006;91:300-6.

15. Noonan JA, Raaijmakers R, Hall BD. Adult height in Noonan syndrome. Am J Med Genet. A 2003;123:68-71.

16. Noordam C, van der Burgt I, Sweep CG, Delemarre-van de Waal HA, Sengers RC, Otten BJ. Growth hormone (GH) secretion in children with Noonan syndrome: frequently abnormal without consequences for growth or response to $\mathrm{GH}$ treatment. Clin Endocrinol (Oxf). 2001;54:53-9.

17. Ahmed ML, Foot AB, Edge JA, Lamkin VA, Savage MO, Dunger DB. Noonan's syndrome: abnormalities of the growth hormone/IGF-I axis and the response to treatment with human biosynthetic growth hormone. Acta Paediatr Scand. 1991;80:446-50.

18. Binder G, Neuer K, Ranke MB, Wittekindt NE. PTPN11 mutations are associated with mild growth hormone resistance in individuals with Noonan syndrome. J Clin Endocrinol Metab. 2005;90:5377-81.

19. Ferreira LV, Souza SA, Arnhold IJ, Mendonca BB, Jorge AA. PTPN11 (protein tyrosine phosphatase, nonreceptor type 11) mutations and response to growth hormone therapy in children with Noonan syndrome. J Clin Endocrinol Metab. 2005;90:5156-60.

20. Sznajer Y, Keren B, Baumann C, Pereira S, Alberti C, Elion J, et al. The spectrum of cardiac anomalies in Noonan syndrome as a result of mutations in the PTPN11 gene. Pediatrics. 2007;119:e1325-31.

21. Shaw AC, Kalidas K, Crosby AH, Jeffery S, Patton MA. The natural history of Noonan syndrome: a long-term follow-up study. Arch Dis Child. 2007;92:128-32.

22. Duncan WJ, Fowler RS, Farkas LG, Ross RB, Wright AW, Bloom $\mathrm{KR}$, et al. A comprehensive scoring system for evaluating Noonan syndrome. Am J Med Genet. 1981;10:37-50.

23. van der Burgt I, Berends E, Lommen E, van Beersum S, Hamel $B$, Mariman E. Clinical and molecular studies in a large Dutch family with Noonan syndrome. Am J Med Genet. 1994;53:187-91.

24. Jamieson $C R$, van der Burgt I, Brady AF, van Reen $M$, Elsawi $M M, H o l ~ F$, et al. Mapping a gene for Noonan syndrome to the long arm of chromosome 12. Nat Genet. 1994;8:357-60.

25. Legius E, Schollen E, Matthijs G, Fryns JP. Fine mapping of Noonan/cardio-facio cutaneous syndrome in a large family. Eur J Hum Genet. 1998;6:32-7.

26. Zenker M, Buheitel G, Rauch R, Koenig R, Bosse K, Kress W, et al. Genotype-phenotype correlations in Noonan syndrome. J Pediatr. 2004;144:368-74.

27. Musante L, Kehl HG, Majewski F, Meinecke P, Schweiger S, Gillessen-Kaesbach G, et al. Spectrum of mutations in PTPN11 and genotype-phenotype correlation in 96 patients with Noonan syndrome and five patients with cardio-facio-cutaneous syndrome. Eur J Hum Genet. 2003;11:201-6.
28. Sarkozy A, Conti E, Seripa D, Digilio MC, Grifone N, Tandoi C, et al. Correlation between PTPN11 gene mutations and congenital heart defects in Noonan and LEOPARD syndromes. $J$ Med Genet. 2003;40:704-8.

29. Bertola DR, Pereira AC, Albano LM, De Oliveira PS, Kim CA, Krieger JE. PTPN11 gene analysis in 74 Brazilian patients with Noonan syndrome or Noonan-like phenotype. Genet Test. 2006;10:186-91.

30. Tartaglia M, Kalidas K, Shaw A, Song X, Musat DL, van der Burgt I, et al. PTPN11 mutations in Noonan syndrome: molecular spectrum, genotype-phenotype correlation, and phenotypic heterogeneity. Am J Hum Genet. 2002;70:1555-63.

31. Tartaglia M, Mehler EL, Goldberg R, Zampino G, Brunner HG, Kremer $\mathrm{H}$, et al. Mutations in PTPN11, encoding the protein tyrosine phosphatase SHP-2, cause Noonan syndrome. Nat Genet. 2001;29:465-8.

32. Schubbert S, Shannon K, Bollag G. Hyperactive ras in developmental disorders and cancer. Nat Rev Cancer. 2007;7: 295-308.

33. Tartaglia M, Martinelli S, Stella L, Bocchinfuso G, Flex E, Cordeddu $\mathrm{V}$, et al. Diversity and functional consequences of germline and somatic PTPN11 mutations in human disease. Am J Hum Genet. 2006;78:279-90.

34. Schubbert S, Bollag G, Shannon K. Deregulated ras signaling in developmental disorders: new tricks for an old dog. Curr Opin Genet Dev. 2007;17:15-22.

35. Pandit B, Sarkozy A, Pennacchio LA, Carta C, Oishi K, Martinelli $S$, et al. Gain-of-function RAF1 mutations cause Noonan and LEOPARD syndromes with hypertrophic cardiomyopathy. Nat Genet. 2007;39:1007-12.

36. Razzaque MA, Nishizawa $T$, Komoike $Y$, Yagi $H$, Furutani $M$, Amo R, et al. Germline gain-of-function mutations in RAF1 cause Noonan syndrome. Nat Genet. 2007;39:1013-7.

37. Tartaglia M, Pennacchio LA, Zhao C, Yadav KK, Fodale V, Sarkozy A, et al. Gain-of-function SOS1 mutations cause a distinctive form of Noonan syndrome. Nat Genet. 2007;39:75-9.

38. Zenker M, Horn D, Wieczorek D, Allanson J, Pauli S, van der Burgt I, et al. SOS1 is the second most common Noonan gene but plays no major role in cardio-facio-cutaneous syndrome. $J$ Med Genet. 2007;44:651-6.

39. Roberts $A E$, Araki $T$, Swanson KD, Montgomery KT, Schiripo TA, Joshi VA, et al. Germline gain-of-function mutations in SOS1 cause Noonan syndrome. Nat Genet. 2007;39:70-4.

40. Nava C, Hanna N, Michot C, Pereira S, Pouvreau N, Niihori T, et al. Cardio-facio-cutaneous and Noonan syndromes due to mutations in the RAS/MAPK signalling pathway: genotypephenotype relationships and overlap with Costello syndrome. J Med Genet. 2007;44:763-71.

41. Schubbert S, Zenker M, Rowe SL, Boll S, Klein C, Bollag G, et al. Germline KRAS mutations cause Noonan syndrome. Nat Genet. 2006;38:331-6.

42. Jorge AA, Marchisotti FG, Montenegro LR, Carvalho LR, Mendonca BB, Arnhold IJ. Growth hormone (GH) pharmacogenetics: influence of $\mathrm{GH}$ receptor exon 3 retention or deletion on first-year growth response and final height in patients with severe GH deficiency. J Clin Endocrinol Metab. 2006;91:1076-80.

43. Ranke MB, Lindberg A, Chatelain P, Wilton P, Cutfield W, Albertsson-Wikland $\mathrm{K}$, et al. Prediction of long-term response to recombinant human growth hormone in Turner syndrome: development and validation of mathematical models. KIGS International Board. Kabi International Growth Study. J Clin Endocrinol Metab. 2000;85:4212-8. 
44. Ranke MB, Lindberg A, Chatelain P, Wilton P, Cutfield W, Albertsson-Wikland K, et al. Derivation and validation of a mathematical model for predicting the response to exogenous recombinant human growth hormone $(\mathrm{GH})$ in prepubertal children with idiopathic GH deficiency. KIGS International Board. Kabi Pharmacia International Growth Study. J Clin Endocrinol Metab. 1999;84:1174-83.

45. Osio D, Dahlgren J, Wikland KA, Westphal O. Improved final height with long-term growth hormone treatment in Noonan syndrome. Acta Paediatr. 2005;94:1232-7.

46. Cotterill AM, McKenna WJ, Brady AF, Sharland M, Elsawi M, Yamada $\mathrm{M}$, et al. The short-term effects of growth hormone therapy on height velocity and cardiac ventricular wall thickness in children with Noonan's syndrome. J Clin Endocrinol Metab. 1996;81:2291-7.
47. GH Research Society. Critical evaluation of the safety of recombinant human growth hormone administration: statement from the Growth Hormone Research Society. J Clin Endocrinol Metab. 2001;86:1868-70.

\section{Endereço para correspondência:}

Alexander A. L. Jorge/Alexsandra C. M. de Moura Ribeiro Laboratório de Hormônios do Hospital das Clínicas Av. Dr. Enéas de Carvalho Aguiar, 155 PAMB, $2^{\circ}$ andar, bloco 6 05403-900, São Paulo, SP.

E-mail: alexj@pq.cnpq.br // aleribeiro@usp.br 